\title{
The efficacy and safety of methylprednisolone in hepatitis B virus- related acute-on-chronic liver failure: a prospective multi-center clinical trial
}

Lin Jia ${ }^{1+}$, Ran Xue ${ }^{1,2 \dagger}$, Yueke Zhu', Juan Zhao ${ }^{1}$, Juan Li', Wei-Ping He ${ }^{3}$, Xiao-Mei Wang ${ }^{4}$, Zhong-Hui Duan', Mei-Xin Ren ${ }^{1}$, Hai-Xia Liu' ${ }^{1}$ Hui-Chun Xing ${ }^{4^{*}}$ and Qing-Hua Meng ${ }^{1 *}$

\begin{abstract}
Background: Hepatitis B virus-related acute-on-chronic liver failure (HBV-ACLF) is a severe condition with high mortality due to lack of efficient therapy. Until now, the use of methylprednisolone (MP) in HBV-ACLF is still controversial. We aimed to evaluate the efficacy and safety of MP in HBV-ACLF.

Methods: Totally 171 HBV-ACLF patients from three medical centers were randomly allocated into MP group (83 patients treated with MP intravenously guttae for 7 days plus standard treatment: $1.5 \mathrm{mg} / \mathrm{kg} /$ day [day 1-3], $1 \mathrm{mg} /$ $\mathrm{kg} /$ day [day 4-5], and $0.5 \mathrm{mg} / \mathrm{kg} /$ day [day 6-7]) and control group (88 patients treated with standard treatment). The primary endpoints were 6 -month mortality and prognostic factors for 6 -month survival. The survival time, cause of death, adverse events, liver function, and HBV DNA replication were analyzed.

Results: The 6-month mortality was significantly lower in MP group than control group [32.4\% vs. 42.5\%, $P=$ 0.0037]. MP treatment was an independent prognostic factor for 6-month survival [HR (95\% Cl) $0.547(0.308-0.973)$; $P=0.040]$. Factors associated with reduced 6-month mortality in MP group included HBV DNA and lymphocyte/ monocyte ratio $(L M R)(P<0.05)$. Based on ROC curve, LMR+MELD had a better predictive value for prognosis of HBV-ACLF under MP treatment. No significant difference in HBV DNA replication was observed between groups $(P>0.05)$.
\end{abstract}

Conclusions: MP therapy is an effective and safe clinical strategy in HBV-ACLF, increasing the 6-month survival rate. Clinical trials registered at http://www.chictr.org.cn as ChiCTR-TRC-13003113 registered on 16 March 2013.

Keywords: Hepatitis B, Acute-on-chronic liver failure, Methylprednisolone

\footnotetext{
*Correspondence: hchxing@sohu.com; meng_qh@126.com

${ }^{\dagger}$ Lin Jia and Ran Xue contributed equally to this work.

${ }^{4}$ Institute of Infectious Diseases, Beijing Di Tan Hospital, Capital Medical

University, No. 8 Jing Shun Dong Street, Chao yang District, Beijing 100015,

China

'Department of Critical Care Medicine of Liver Disease, Beijing You-An

Hospital, Capital Medical University, Beijing, China

Full list of author information is available at the end of the article
}

C C The Author(s). 2020 Open Access This article is licensed under a Creative Commons Attribution 4.0 International License, which permits use, sharing, adaptation, distribution and reproduction in any medium or format, as long as you give appropriate credit to the original author(s) and the source, provide a link to the Creative Commons licence, and indicate if changes were made. The images or other third party material in this article are included in the article's Creative Commons licence, unless indicated otherwise in a credit line to the material. If material is not included in the article's Creative Commons licence and your intended use is not permitted by statutory regulation or exceeds the permitted use, you will need to obtain permission directly from the copyright holder. To view a copy of this licence, visit http://creativecommons.org/licenses/by/4.0/. The Creative Commons Public Domain Dedication waiver (http://creativecommons.org/publicdomain/zero/1.0/) applies to the data made available in this article, unless otherwise stated in a credit line to the data. 


\section{Background}

In Asia, hepatitis B virus-related acute-on-chronic liver failure (HBV-ACLF) accounts for about $70 \%$ of all ACLF cases, which is identified with severe acute exacerbation (AE) of liver function to liver failure in the chronic hepatitis $\mathrm{B}(\mathrm{CHB})$ patients $[1,2]$, with high mortality (51.6 to $54.3 \%$ ) [3]. Liver transplantation remains the only curative treatment for ACLF with limited application [4-6]. Up to now, there is no effective treatment which has been developed for HBV-ACLF patients.

Overwhelming systemic inflammation and susceptibility to infection are two key features of ACLF $[7,8]$. "Endotoxin-macrophage-cytokine storm" is the core pathogenesis of liver failure. The chemical essence of endotoxin is lipopolysaccharide (LPS) [9]. With the interaction of LPS-binding protein, it binds to a variety of cell membrane receptor, stimulating the synthesis and release of cytokines, involving interferon- $\alpha$ (INF- $\alpha$ ) and IL-12. As the most commonly used immunosuppressive and anti-inflammatory agent, methylprednisolone (MP) has theoretical basis for the treatment of ACLF [10-12]. However, until now, the use of methylprednisolone (MP) in HBV-ACLF is still uncertain and controversial [13].

With the coming of nucleoside analogs (NAs), more and more guidelines have recommended NAs to be used in patients with acute exacerbation of chronic HBV infection. The early combined use of NAs and MP could be a good option to reverse the potential deterioration in patients with HBV-related liver failure. Our previous study has demonstrated that MP can improve 28-day survival rate in HBV-ACLF patients [14]. A recent study has also reported that early combination therapy with glucocorticoids (GCs) and NAs induces rapid resolution of inflammation in ALF due to transient HBV infection [15]. However, Huang $\mathrm{C}$ et al. [16] investigated retrospectively the efficacy of GCs in patients with HBVACLF, which indicated that GCs treatment did not improve transplant-free survival in patients with $\mathrm{HBV}$ ACLF.

Therefore, in order to further evaluate the efficacy and safety of MP in HBV-ACLF patients, we proceed this multi-center, prospective randomized controlled clinical trial to provide evidence for MP as one of the clinically effective treatments for HBV-ACLF.

\section{Methods}

Eligibility

Patients were recruited at Beijing Di Tan Hospital, Capital Medical University, People's Liberation Army No. 302 Hospital, and Beijing You-An Hospital, Capital Medical University, from April 2013 to May 2015. All procedures related to this research were accorded morally with current laws as well as the creeds of the
Declaration of Helsinki. The research was permitted by the Ethical Committee of Beijing You-An Hospital, Capital Medical University, Beijing Di Tan Hospital Capital Medical University, and People's Liberation Army No. 302 Hospital (No.2 [2013]). All study participants gave their informed consent to participate in the study. The process of study selection and exclusion was shown in Fig. 1.

\section{Patients selection}

The inclusion criteria were the following: (1) aged 18 years or older; (2) previously diagnosed or undiagnosed HBV, HBsAg positive; and (3) all enrolled patients met the criteria for ACLF from the consensus recommendations of the Asian Pacific Association for the Study of the Liver (APASL) specified as follows: an acute hepatic insult manifesting as jaundice (serum bilirubin $\geq 5 \mathrm{mg} / \mathrm{dl}$ [ $\geq 85 \mu \mathrm{mol} / \mathrm{l}]$ ) and coagulopathy (INR $\geq 1.5$ or prothrombin activity $\leq 40 \%$ ) complicated within 4 weeks by clinical ascites and/or encephalopathy in a patient with previously diagnosed or undiagnosed chronic liver disease/cirrhosis [17, 18]. Diagnostic criteria for cirrhosis are made by history, physical examination, and previously available laboratory, fibrosis biomarkers (e.g., FIB4 or FibroTest), endoscopic or radiologic investigations (ultrasound, CT abdomen or transient elastography [fibroscan]), or a previously liver biopsy history [19].

The exclusion criteria comprised the following: (1) uncontrolled bacterial infection or gastrointestinal hemorrhage before enrollment; (2) infection with hepatitis virus other than HBV, or human immunodeficiency virus; (3) autoimmune diseases, alcoholic liver disease, and drug-induced hepatitis; and (4) serious renal, cardiac, respiratory, neurologic diseases, or any detectable tumor.

The diagnostic criteria of complications included (1) gastrointestinal hemorrhage confirmed by endoscopy, (2) bacterial infection diagnosed by a positive culture result [20], (3) fungal infection diagnosed according to EORTC/MSG definition [21], (4) hepatorenal syndrome (HRS) diagnosed according to the International Ascites Club's guidelines [22], (5) spontaneous bacterial peritonitis (SBP) diagnosed based on diagnostic paracentesis [23], (6) electrolyte disturbance defined as $\geq 1$ electrolyte abnormalities of $\mathrm{K}+, \mathrm{Na}+$, and $\mathrm{Cl}-$, (7) hypoalbuminemia diagnosed when albumin $<35 \mathrm{~g} / \mathrm{L}$, and (8) pleural effusion diagnosed by $\mathrm{X}$ rays or computerized tomography.

\section{Study design}

After investigators confirmed eligibility, patients were randomized (1:1 allocation ratio) to MP plus standard management (MP group) or standard treatments (control group) by computer-generated permutated block randomization (block size of four) stratified. Treatment 


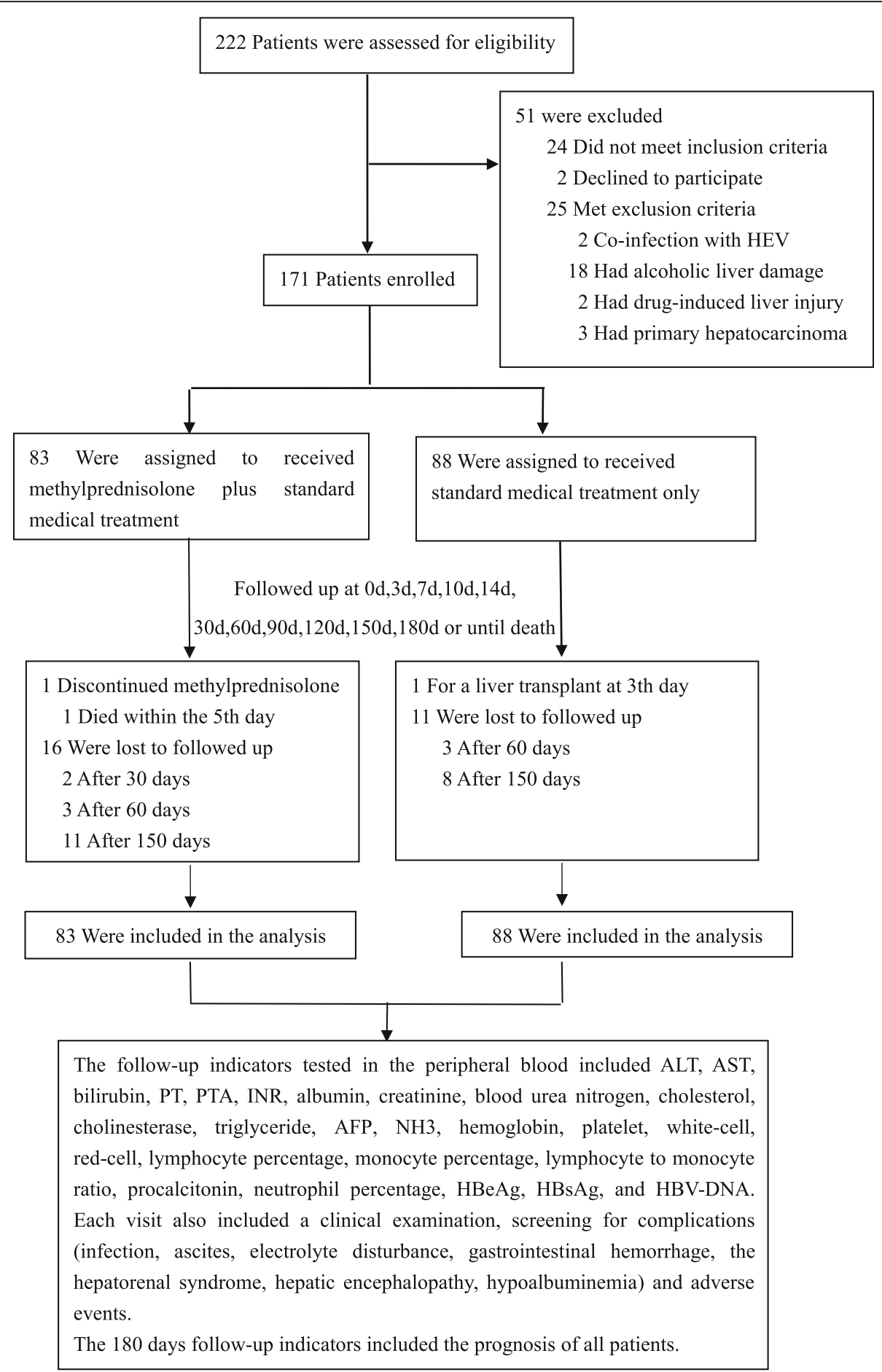

Fig. 1 Flowchart for the enrollment of patients with HBV-ACLF

was started when patients were enrolled and the day of enrollment was defined as day 1 .

Patients were followed up at the baseline ( 0 days), 3 days, 7 days, 10 days, 14 days, 30 days, and then monthly until the 6th month. Collected information included laboratory tests, screening for complications. The alive status means significant improvement in clinical symptoms, bilirubin $<$ $5 \times$ upper limit of normal (5ULN), PTA $>30 \%$, or INR $<1.5$.

\section{Treatment protocols}

Standard treatments included antiviral drugs (lamivudine $100 \mathrm{mg} /$ day, adefovir dipivoxil $10 \mathrm{mg} /$ day, entecavir 0.5 
Table 1 Baseline characteristics and complications of patients

\begin{tabular}{|c|c|c|c|c|}
\hline Characteristic & No. of patients $(N=171)$ & Methylprednisolone $(N=83)$ & Control $(N=88)$ & $P$ \\
\hline$\overline{\text { Male }^{a}}$ & $152(88.9 \%)$ & $74(8.4 \%)$ & $78(88.6 \%)$ & 0.914 \\
\hline Age (years) & $45.2 \pm 12.3$ & $43.7 \pm 12.7$ & $46.6 \pm 11.8$ & 0.121 \\
\hline Cirrhosis $^{a}$ & 139 (81.3\%) & 67 (80.7\%) & 72 (81.8\%) & 0.854 \\
\hline Time from onset ${ }^{d}$ to admission (days) ${ }^{b}$ & $20(10-30)$ & $16(10-21)$ & $20(13-30)$ & 0.083 \\
\hline MELD score & $23.3 \pm 4.7$ & $22.8 \pm 4.0$ & $23.9 \pm 5.2$ & 0.132 \\
\hline Lg HBV DNA (IU/ml) & $4.8 \pm 1.9$ & $4.9 \pm 2.0$ & $4.6 \pm 1.8$ & 0.383 \\
\hline \multicolumn{5}{|l|}{ HBV DNA ${ }^{a}$} \\
\hline Positive & $150(87.7 \%)$ & $70(84.3 \%)$ & $80(90.9 \%)$ & 0.191 \\
\hline Negative & $21(12.3 \%)$ & $13(15.7 \%)$ & $8(9.1 \%)$ & 0.191 \\
\hline \multicolumn{5}{|l|}{$\mathrm{HBeAg}^{\mathrm{a}}$} \\
\hline Positive & $90(52.6 \%)$ & $43(51.8 \%)$ & $47(53.4 \%)$ & 0.834 \\
\hline Negative & $81(47.4 \%)$ & $40(48.2 \%)$ & $41(46.6 \%)$ & 0.834 \\
\hline $\mathrm{HBsAg}{ }^{\mathrm{b}}$ & $2582.0(401.0-6012.0)$ & $2582.0(422.8-5884.0)$ & $2613.0(325.4-6197.3)$ & 0.843 \\
\hline No medication with NAs when the disease onset ${ }^{a}$ & $127(74.3 \%)$ & $58(69.9 \%)$ & $69(78.4 \%)$ & 0.202 \\
\hline Never used before & $89(52.0 \%)$ & $38(45.8 \%)$ & $51(58.0 \%)$ & \\
\hline Drug withdrawal & $38(22.2 \%)$ & $20(24.1 \%)$ & $18(20.5 \%)$ & \\
\hline ADV & $6(3.5 \%)$ & $2(2.4 \%)$ & $4(4.5 \%)$ & \\
\hline ETV & $16(9.4 \%)$ & $12(14.5 \%)$ & $4(4.5 \%)$ & \\
\hline LAM & $10(5.8 \%)$ & $4(4.8 \%)$ & $6(6.8 \%)$ & \\
\hline ETV+ADV & $3(1.8 \%)$ & $1(1.2 \%)$ & $2(2.3 \%)$ & \\
\hline LAM+ADV & $3(1.8 \%)$ & $1(1.2 \%)$ & $2(2.3 \%)$ & \\
\hline Medication with NAs when the disease onset ${ }^{a}$ & $44(25.7 \%)$ & $25(30.1 \%)$ & $19(21.6 \%)$ & 0.202 \\
\hline LAM & $7(4.1 \%)$ & $4(4.8 \%)$ & $3(3.4 \%)$ & \\
\hline LAM+TDF & $1(0.6 \%)$ & $1(1.2 \%)$ & 0 & \\
\hline LAM+ADV & $7(4.1 \%)$ & $3(3.6 \%)$ & $4(4.5 \%)$ & \\
\hline ETV & $26(15.2 \%)$ & $16(19.3 \%)$ & $10(11.4 \%)$ & \\
\hline ADV & $2(1.2 \%)$ & 0 & $2(2.3 \%)$ & \\
\hline LdT & $1(0.6 \%)$ & $1(1.2 \%)$ & 0 & \\
\hline NAs therapy after enrollment ${ }^{a}$ & $171(100 \%)$ & $83(100 \%)$ & 88 (100\%) & 0.101 \\
\hline LAM & $16(9.4 \%)$ & $6(7.2 \%)$ & $10(11.4 \%)$ & \\
\hline LAM+ADV & $7(4.1 \%)$ & $5(6.0 \%)$ & $2(2.3 \%)$ & \\
\hline LAM+TDF & $1(0.6 \%)$ & $1(1.2 \%)$ & 0 & \\
\hline ETV & $142(83.0 \%)$ & $69(83.1 \%)$ & $73(83.0 \%)$ & \\
\hline ETV+ADV & $2(1.2 \%)$ & 0 & $2(2.3 \%)$ & \\
\hline ADV & $1(0.6 \%)$ & 0 & $1(1.1 \%)$ & \\
\hline LdT & $2(1.2 \%)$ & $2(2.4 \%)$ & 0 & \\
\hline $\operatorname{ALT}(U / L)^{b}$ & $307.0(117.2-573.6)$ & $347.0(131.7-685.9)$ & $276.3(106.8-514.1)$ & 0.080 \\
\hline $\operatorname{AST}(U / L)^{b}$ & $214.0(156.8-485.0)$ & $291.3(156.8-515.0)$ & $184.4(155.5-306.1)$ & 0.054 \\
\hline Bilirubin ( $\mu \mathrm{mol} / \mathrm{L})$ & $353.9 \pm 138.4$ & $335.3 \pm 133.8$ & $371.4 \pm 141.2$ & 0.089 \\
\hline Albumin (g/L) & $31.0 \pm 4.1$ & $31.4 \pm 3.6$ & $30.7 \pm 4.5$ & 0.257 \\
\hline Cholinesterase (IU/L) & $3215.8 \pm 1165.5$ & $3262.0 \pm 1063.3$ & $3172.3 \pm 1258.9$ & 0.616 \\
\hline $\operatorname{AFP}(n g / m l)^{b}$ & $80.0(33.1-180.0)$ & $86.0(45.5-240.0)$ & $65.7(21.7-132.8)$ & 0.150 \\
\hline Cholesterol (mmol/L) & $2.3 \pm 0.8$ & $2.4 \pm 0.9$ & $2.2 \pm 0.7$ & 0.059 \\
\hline Blood urea nitrogen $(\mathrm{mmol} / \mathrm{L})^{b}$ & $4.0(3.2-6.1)$ & $4.6(3.1-6.1)$ & $4.3(3.5-6.3)$ & 0.054 \\
\hline
\end{tabular}


Table 1 Baseline characteristics and complications of patients (Continued)

\begin{tabular}{|c|c|c|c|c|}
\hline Characteristic & No. of patients $(N=171)$ & Methylprednisolone $(N=83)$ & Control $(N=88)$ & $P$ \\
\hline Creatinine $(\mu \mathrm{mol} / \mathrm{L})^{\mathrm{b}}$ & $66.2(56.8-81.0)$ & $64.4(55.4-76.6)$ & $68.0(58.1-84.5)$ & 0.123 \\
\hline $\mathrm{NH} 3(\mu \mathrm{g} / \mathrm{dl})$ & $86.5 \pm 45.2$ & $89.2 \pm 44.9$ & $84.0 \pm 45.7$ & 0.449 \\
\hline Triglyceride $(\mathrm{mmol} / \mathrm{L})^{\mathrm{b}}$ & $0.9(0.6-1.5)$ & $0.8(0.5-1.7)$ & $0.9(0.6-1.4)$ & 0.936 \\
\hline White blood cell $\left(\times 10^{9} / \mathrm{L}\right)$ & $7.3 \pm 3.5$ & $7.3 \pm 3.4$ & $7.3 \pm 3.6$ & 0.960 \\
\hline Lymphocyte percentage & $20.7 \pm 8.5$ & $20.1 \pm 8.1$ & $21.3 \pm 8.9$ & 0.372 \\
\hline Monocyte percentage & $9.5 \pm 3.6$ & $9.0 \pm 3.5$ & $9.8 \pm 3.7$ & 0.147 \\
\hline Lymphocyte/monocyte ratio ${ }^{\mathrm{b}}$ & $2.2(1.5-3.0)$ & $2.2(1.5-3.0)$ & $2.1(1.5-2.9)$ & 0.502 \\
\hline Neutrophil percentage & $68.6 \pm 10.6$ & $69.1 \pm 9.6$ & $68.1 \pm .11 .4$ & 0.547 \\
\hline Procalcitonin $(\mu \mathrm{g} / L)^{b}$ & $0.6(0.4-0.9)$ & $0.6(0.5-1.0)$ & $0.6(0.3-0.8)$ & 0.197 \\
\hline PT $(s)^{b}$ & $22.9(20.2-27.9)$ & $22.9(19.2-22.8)$ & $22.9(20.7-27.6)$ & 0.877 \\
\hline PTA (\%) & $35.9 \pm 9.2$ & $36.0 \pm .10 .3$ & $35.8 \pm 8.1$ & 0.865 \\
\hline$I_{N R}{ }^{a}$ & $2.9(1.7-2.3)$ & $2.1(1.6-2.6)$ & $2.0(1.7-2.2)$ & 0.580 \\
\hline Red blood cell $\left(\times 10^{12} / \mathrm{L}\right)$ & $3.9 \pm 0.8$ & $4.0 \pm 0.8$ & $3.8 \pm 0.9$ & 0.070 \\
\hline Hemoglobin (g/L) & $124.2 \pm 21.1$ & $127.6 \pm 18.9$ & $121.5 \pm 22.3$ & 0.056 \\
\hline Platelet $\left(\times 10^{9} / L\right)$ & $105.2 \pm 51.6$ & $113.0 \pm 45.3$ & $97.9 \pm 56.1$ & 0.055 \\
\hline Ascites $^{a}$ & $103(60.2 \%)$ & $45(54.2 \%)$ & $58(65.9 \%)$ & 0.118 \\
\hline Infections $^{\mathrm{a}}$ & $92(53.8 \%)$ & $47(56.6 \%)$ & $45(51.1 \%)$ & 0.472 \\
\hline Electrolyte disturbance $^{a}$ & 17 (9.9\%) & $5(6.0 \%)$ & $12(13.6 \%)$ & 0.096 \\
\hline Encephalopathy ${ }^{a}$ & $21(12.3 \%)$ & $12(14.5 \%)$ & $9(10.2 \%)$ & 0.400 \\
\hline Hypoalbuminemia $^{a}$ & $35(20.5 \%)$ & $16(19.3 \%)$ & $19(21.6 \%)$ & 0.708 \\
\hline Hepatorenal syndrome ${ }^{a}$ & $5(2.9 \%)$ & $1(1.2 \%)$ & $4(4.5 \%)$ & 0.369 \\
\hline Pleural effusion ${ }^{a}$ & $7(4.1 \%)$ & $3(3.6 \%)$ & $4(4.5 \%)$ & 1.000 \\
\hline Hyperthyroidism $^{a}$ & $1(0.6 \%)$ & $1(1.2 \%)$ & 0 & 0.485 \\
\hline Peptic ulcer ${ }^{a}$ & $2(1.2 \%)$ & $1(1.2 \%)$ & $1(1.1 \%)$ & 1.000 \\
\hline Artificial liver support after enrollment ${ }^{c}$ & - & 87.98 & 84.13 & 0.582 \\
\hline Artificial centesis after enrollment ${ }^{c}$ & - & 79.55 & 92.08 & 0.090 \\
\hline The time of antibiotic use after enrollment ${ }^{c}$ & - & 91.72 & 80.60 & 0.141 \\
\hline
\end{tabular}

${ }^{\mathrm{a}}$ Data expressed with number of cases

${ }^{b}$ Data expressed as median (range)

'Date expressed with mean rank

${ }^{\mathrm{d} O n s e t:}$ appearance of nausea, poor appetite, jaundice, or gastrointestinal hemorrhage

$\mathrm{mg}$ /day, telbivudine $600 \mathrm{mg} /$ day, or tenofovir disoproxil fumarate $300 \mathrm{mg} /$ day based on individual's condition before enrollment), nutritional support (1.5-2.0 g protein/ $\mathrm{kg} /$ day and $35-40 \mathrm{kcal} / \mathrm{kg} /$ day), plasma exchange, and complications control such as anti-infection, administration of human serum albumin ( $10 \mathrm{~g}$ per day until serum albumin was $35 \mathrm{~g} / \mathrm{L})$, fresh frozen plasma $(200 \mathrm{ml}$ to 400 $\mathrm{ml} /$ day until the INR $<1.5$ ), and vasoactive agents (terlipressin alone or in combination with noradrenaline to reverse septic shock). All treatments were performed based on the criteria of diagnostic and treatment guidelines for ACLF adopted by the Chinese Medical Association [24].

The MP group was given standard treatments combined with MP intravenously guttae for 7 days: $1.5 \mathrm{mg} /$ $\mathrm{kg} /$ day, days $1-3$; thereafter $1 \mathrm{mg} / \mathrm{kg} /$ day, days $4-5$; and followed by $0.5 \mathrm{mg} / \mathrm{kg} /$ day, days $6-7$.

\section{Clinical and laboratory parameters}

Laboratory tests measured PTA, prothrombin time (PT), INR, bilirubin, aspartate aminotransferase (AST), alanine aminotransferase (ALT), albumin, creatinine, blood urea nitrogen, cholesterol, cholinesterase, triglyceride, alphafatal protein (AFP), blood ammonia, hemoglobin, platelet, white blood cell, red blood cell, lymphocyte percentage, monocyte percentage, lymphocyte to monocyte ratio (LMR), and procalcitonin and neutrophil percentage with routine automated techniques. HBsAg and $\mathrm{HBeAg}$ were assayed using commercially available radioimmunoassay kits (Roche Diagnostics). Serum HBVDNA was quantified using a cross linking chemical hybridization assay (Roche Diagnostics) and the detection threshold is 100 copies $/ \mathrm{ml}$. All hospitals use the same assay and standard operating procedure for the 


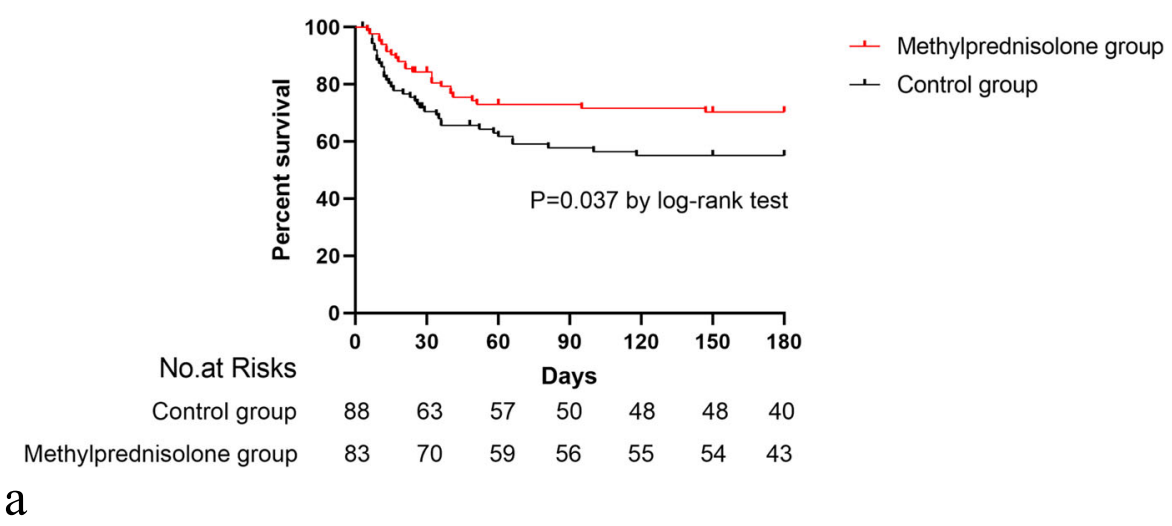

The mortality during different time points

\begin{tabular}{llllll}
\hline & $\begin{array}{l}\text { Methylprednisolone } \\
\text { group(N=83) }\end{array}$ & $\begin{array}{l}\text { Control group } \\
(\mathrm{N}=88)\end{array}$ & $\mathrm{P}$ & $\begin{array}{l}\text { Hazard } \\
\text { Ratio }\end{array}$ & 95\%CI \\
\hline $14 \mathrm{~d}$ & $8.4 \%(7 / 83)$ & $23.9 \%(21 / 88)$ & 0.007 & 2.814 & $1.335-5.930$ \\
$30 \mathrm{~d}$ & $16.9 \%(14 / 83)$ & $35.2 \%(31 / 88)$ & 0.006 & 2.294 & $1.275-4.129$ \\
$60 \mathrm{~d}$ & $28.4 \%(23 / 81)$ & $43.2 \%(38 / 88)$ & 0.017 & 1.841 & $1.116-3.037$ \\
$90 \mathrm{~d}$ & $29.5 \%(23 / 78)$ & $49.4 \%(42 / 85)$ & 0.006 & 1.983 & $1.216-3.234$ \\
$120 \mathrm{~d}$ & $30.8 \%(24 / 78)$ & $51.8 \%(44 / 85)$ & 0.004 & 2.004 & $1.242-3.232$ \\
$150 \mathrm{~d}$ & $32.1 \%(25 / 78)$ & $51.8 \%(44 / 85)$ & 0.007 & 1.934 & $1.203-3.109$ \\
$180 \mathrm{~d}$ & $37.3 \%(25 / 67)$ & $58.4 \%(45 / 77)$ & 0.005 & 1.979 & $1.235-3.171$ \\
\hline
\end{tabular}

b

The causes-of-death in different groups

\begin{tabular}{lccccc}
\hline Causes & $\begin{array}{c}\text { Methylpredni } \\
\text { solone group } \\
\%(\mathrm{~N})\end{array}$ & $\begin{array}{c}\text { Control } \\
\text { group } \\
\%(\mathrm{~N})\end{array}$ & $\mathrm{P}$ & $\begin{array}{c}\text { Odds } \\
\text { Ratio }\end{array}$ & 95\%CI \\
\hline Infection & $10.4 \%(7)$ & $15.6 \%(12)$ & 0.364 & 0.632 & $0.233-1.711$ \\
HRS & $10.4 \%(7)$ & $11.7 \%(9)$ & 0.813 & 0.881 & $0.309-2.511$ \\
HE & $9.0 \%(6)$ & $9.1 \%(7)$ & 0.977 & 0.984 & $0.314-3.085$ \\
MOF & $1.5 \%(1)$ & $5.2 \%(4)$ & 0.451 & 0.277 & $0.030-2.537$ \\
gastrointestinal & $3.0 \%(2)$ & $5.2 \%(4)$ & 0.807 & 0.562 & $0.100-3.167$ \\
hemorrhage & & & & & \\
cerebral & $1.5 \%(1)$ & $1.3 \%(1)$ & 1.000 & 1.152 & $0.071-18.773$ \\
hemorrhage & & & & & \\
\hline $\mathrm{C}$
\end{tabular}

Fig. 2 a A significant survival advantage was observed during 6 months among patients who received methylprednisolone. b The mortality during different time. $\mathbf{c}$ The causes-of-death in different groups

above indicators. Model for end-stage liver disease (MELD) score was calculated according to the original formula proposed by the Mayo Clinic [25].

\section{Study outcomes}

The primary endpoints were 6-month mortality and prognostic factors for 6-month survival. The secondary endpoints were adverse events and changes of laboratory indices during treatment.

\section{Statistical analysis}

Quantitative variables, expressed as means \pm SD or medians (interquartile range), were compared using Wilcoxon, Kruskal-Wallis, or Student's $t$ tests, as appropriate. Qualitative variables, expressed as percentages, 
Table 2 Analysis of factors associated with mortality at 6 months

\begin{tabular}{|c|c|c|c|c|}
\hline \multirow[t]{2}{*}{ Factors } & \multicolumn{2}{|l|}{ Univariate } & \multicolumn{2}{|l|}{ Multivariate } \\
\hline & $\mathrm{HR}(95 \% \mathrm{Cl})$ & $\overline{P \text { value }}$ & $\mathrm{HR}(95 \% \mathrm{Cl})$ & $P$ value \\
\hline Methylprednisolone & $0.584(0.349-0.976)$ & 0.040 & $0.547(0.308-0.973)$ & 0.040 \\
\hline Centers $^{a}$ & - & 0.683 & & \\
\hline Cirrhosis & $1.114(0.566-2.194)$ & 0.755 & & \\
\hline Antiviral treatment ${ }^{a}$ & - & 0.410 & & \\
\hline Male & $0.594(0.293-1.205)$ & 0.149 & & \\
\hline Age (years) & $1.024(1.004-1.045)$ & 0.019 & $1.012(0.987-1.037)$ & 0.359 \\
\hline Time from onset to admission (days) & $1.008(0.993-1.024)$ & 0.304 & & \\
\hline MELD score & $1.123(1.072-1.176)$ & 0.000 & & \\
\hline Ig HBV DNA (IU/ml) & $0.973(0.851-1.112)$ & 0.686 & & \\
\hline HBV DNA positive & $0.617(0.313-1.218)$ & 0.164 & & \\
\hline HBeAg positive & $0.726(0.439-1.201)$ & 0.213 & & \\
\hline $\mathrm{HBsAg}$ & $1.000(1.000-1.000)$ & 0.248 & & \\
\hline $\mathrm{ALT}(\mathrm{U} / \mathrm{L})$ & $0.999(0.999-1.000)$ & 0.092 & & \\
\hline AST(U/L) & $1.000(0.999-1.001)$ & 0.859 & & \\
\hline Bilirubin ( $\mu \mathrm{mol} / \mathrm{L})$ & $1.004(1.002-1.005)$ & 0.000 & $1.003(1.001-1.005)$ & 0.004 \\
\hline Albumin(g/L) & $0.985(0.924-1.050)$ & 0.648 & & \\
\hline Cholinesterase (IU/L) & $1.000(1.000-1.000)$ & 0.893 & & \\
\hline $\operatorname{AFP}(\mathrm{ng} / \mathrm{ml})$ & $0.999(0.997-1.001)$ & 0.228 & & \\
\hline Blood urea nitrogen (mmol/L) & $1.149(1.049-1.260)$ & 0.003 & $1.195(1.018-1.402)$ & 0.029 \\
\hline Creatinine $(\mu \mathrm{mol} / \mathrm{L})$ & $1.006(1.001-1.011)$ & 0.020 & $0.995(0.984-1.005)$ & 0.318 \\
\hline $\mathrm{NH} 3(\mu \mathrm{g} / \mathrm{dl})$ & $1.002(0.997-1.008)$ & 0.413 & & \\
\hline Triglyceride (mmol/L) & $0.628(0.401-0.986)$ & 0.043 & $0.905(0.524-1.561)$ & 0.719 \\
\hline Cholesterol (mmol/L) & $0.835(0.585-1.190)$ & 0.319 & & \\
\hline White blood cell $\left(\times 10^{9} / \mathrm{L}\right)$ & $1.057(0.986-1.133)$ & 0.115 & & \\
\hline Lymphocyte percentage & $0.934(0.903-0.966)$ & 0.000 & $0.950(0.883-1.023)$ & 0.175 \\
\hline Monocyte percentage & $1.103(1.023-1.190)$ & 0.011 & $1.149(1.011-1.307)$ & 0.034 \\
\hline Lymphocyte to monocyte ratio & $0.497(0.366-0.674)$ & 0.000 & $0.838(0.468-1.502)$ & 0.553 \\
\hline Neutrophil percentage & $1.037(1.012-1.062)$ & 0.003 & & \\
\hline Procalcitonin ( $\mu \mathrm{g} / \mathrm{L})$ & $0.993(0.871-1.132)$ & 0.913 & & \\
\hline PTA (\%) & $0.942(0.914-0.970)$ & 0.000 & $0.931(0.870-0.996)$ & 0.038 \\
\hline INR & $2.272(1.482-3.493)$ & 0.000 & $0.918(0.320-2.629)$ & 0.873 \\
\hline Red blood cell $\left(\times 10^{12} / \mathrm{L}\right)$ & $0.959(0.711-1.293)$ & 0.782 & & \\
\hline Hemoglobin(g/L) & $0.998(0.986-1.009)$ & 0.702 & & \\
\hline Platelet $\left(\times 10^{9} / \mathrm{L}\right)$ & $0.996(0.991-1.002)$ & 0.163 & & \\
\hline Ascites & $2.021(1.154-3.539)$ & 0.014 & $0.879(0.467-1.656)$ & 0.690 \\
\hline Infections & $1.381(0.826-2.308)$ & 0.218 & & \\
\hline Electrolyte disturbance & $0.599(0.217-1.651)$ & 0.322 & & \\
\hline $\mathrm{HE}$ & $2.161(1.148-4.066)$ & 0.017 & $1.270(0.629-2.564)$ & 0.505 \\
\hline Hypoalbuminemia & $1.010(0.537-1.900)$ & 0.975 & & \\
\hline HRS & 4.296 (1.544-11.954) & 0.005 & $1.013(0.216-4.753)$ & 0.987 \\
\hline
\end{tabular}


Table 3 Association between different factors and 6-month mortality in the methylprednisolone group

\begin{tabular}{|c|c|c|c|c|}
\hline \multirow[t]{2}{*}{ Factors } & \multicolumn{2}{|l|}{ Univariate } & \multicolumn{2}{|l|}{ Multivariate } \\
\hline & $\mathrm{HR}(95 \% \mathrm{Cl})$ & $P$ & HR $(95 \% \mathrm{Cl})$ & $P$ \\
\hline Male & $0.496(0.169-1.451)$ & 0.200 & & \\
\hline Centers $^{a}$ & - & 0.575 & & \\
\hline Antiviral treatment ${ }^{a}$ & - & 0.681 & & \\
\hline Age (years) & $1.006(0.975-1.039)$ & 0.695 & & \\
\hline cirrhosis & $1.254(0.429-3.670)$ & 0.679 & & \\
\hline Time from onset to admission (days) & $1.021(1.001-1.043)$ & 0.044 & $1.021(0.997-1.045)$ & 0.085 \\
\hline MELD scores & $1.144(1.053-1.244)$ & 0.001 & & \\
\hline Ig HBV DNA & $0.812(0.653-1.008)$ & 0.060 & & \\
\hline HBV DNA positive & $0.247(0.105-0.584)$ & 0.001 & $4.875(1.596-14.889)$ & 0.005 \\
\hline HBeAg positive & $1.130(0.506-2.523)$ & 0.765 & & \\
\hline $\mathrm{HBsAg}$ & $1.000(1.000-1.000)$ & 0.184 & & \\
\hline $\mathrm{ALT}(\mathrm{U} / \mathrm{L})$ & $0.999(0.999-1.000)$ & 0.213 & & \\
\hline AST(U/L) & $1.000(0.999-1.001)$ & 0.669 & & \\
\hline Bilirubin $(\mu \mathrm{mol} / \mathrm{L})$ & $1.005(1.002-1.008)$ & 0.001 & $1.001(0.998-1.004)$ & 0.534 \\
\hline Cholinesterase (IU/L) & $1.000(0.999-1.000)$ & 0.375 & & \\
\hline AFP (ng/ml) & $0.997(0.994-1.001)$ & 0.111 & & \\
\hline Albumin $(\mathrm{g} / \mathrm{L})$ & $0.978(0.872-1.096)$ & 0.700 & & \\
\hline Blood urea nitrogen $(\mathrm{mmol} / \mathrm{L})$ & $1.094(0.941-1.271)$ & 0.241 & & \\
\hline Creatinine $(\mu \mathrm{mol} / \mathrm{L})$ & $1.000(0.987-1.013)$ & 0.976 & & \\
\hline $\mathrm{NH} 3(\mu \mathrm{mol} / \mathrm{L})$ & $1.005(0.998-1.012)$ & 0.152 & & \\
\hline Triglyceride $(\mathrm{mmol} / \mathrm{L})$ & $0.731(0.394-1.356)$ & 0.320 & & \\
\hline Cholesterol (mmol/L) & $0.896(0.537-1.496)$ & 0.675 & & \\
\hline White cell $\left(\times 10^{9} / L\right)$ & $1.049(0.942-1.168)$ & 0.381 & & \\
\hline Red cell $\left(\times 10^{9} / \mathrm{L}\right)$ & $0.996(0.611-1.622)$ & 0.986 & & \\
\hline Hemoglobin (g/L) & $0.998(0.978-1.019)$ & 0.870 & & \\
\hline Platelet $\left(\times 10^{9} / \mathrm{L}\right)$ & $0.999(0.990-1.008)$ & 0.851 & & \\
\hline Lymphocyte percentage & $0.955(0.904-1.008)$ & 0.096 & & \\
\hline Monocyte percentage & 1.181 (1.049-1.329) & 0.006 & $1.156(0.954-1.401)$ & 0.138 \\
\hline Lymphocyte/monocyte ratio & $0.533(0.339-0.839)$ & 0.007 & $0.537(0.291-0.992)$ & 0.047 \\
\hline Procalcitonin $(\mu \mathrm{g} / \mathrm{L})$ & $0.992(0.732-1.344)$ & 0.957 & & \\
\hline Neutrophil percentage & $1.018(0.978-1.060)$ & 0.375 & & \\
\hline PTA (\%) & $0.929(0.887-0.974)$ & 0.002 & $0.901(0.790-1.028)$ & 0.122 \\
\hline INR & $2.764(1.401-5.451)$ & 0.003 & $0.613(0.074-5.075)$ & 0.650 \\
\hline Ascites & $2.467(1.021-5.959)$ & 0.045 & $1.143(0.398-3.276)$ & 0.804 \\
\hline Infections & $1.344(0.588-3.072)$ & 0.484 & & \\
\hline Electrolyte disturbance & $0.045(0.000-64.307)$ & 0.403 & & \\
\hline $\mathrm{HE}$ & $3.257(1.347-7.873)$ & 0.009 & $2.746(0.868-8.683)$ & 0.085 \\
\hline Hypoalbuminemia & $1.183(0.404-3.464)$ & 0.759 & & \\
\hline HRS & $6.965(0.899-53.954)$ & 0.063 & & \\
\hline
\end{tabular}

${ }^{a}$ Risk estimate statistics cannot be computed. They are only computed for two groups of data

were compared using chi-square or Fisher's exact tests. Ordinal enumeration variables were analyzed by rank sum tests. The changes of indices during follow up time were compared using variance analysis for repeated data. Kaplan-Meier survival curves were plotted and compared with log-rank tests. Significantly predictive factors 
Table 4 Collinearity diagnostic for all indexes which included in multivariate Cox analysis in HBV-ACLF patients

\begin{tabular}{|c|c|c|c|c|c|}
\hline \multirow[t]{2}{*}{ Model } & \multicolumn{2}{|c|}{ Collinearity statistics } & \multirow[t]{2}{*}{ Model } & \multicolumn{2}{|c|}{ Collinearity statistics } \\
\hline & Tolerance & VIF & & Tolerance & VIF \\
\hline Age & 0.814 & 1.228 & & 0.852 & 1.174 \\
\hline Male & 0.833 & 1.200 & & 0.863 & 1.159 \\
\hline Methylprednisolone & 0.856 & 1.168 & & 0.856 & 1.168 \\
\hline MELD & 0.048 & 20.674 & & - & - \\
\hline ALT & 0.778 & 1.285 & & 0.787 & 1.270 \\
\hline TBIL & 0.324 & 3.088 & & 0.772 & 1.295 \\
\hline Blood urea nitrogen & 0.504 & 1.986 & & 0.504 & 1.986 \\
\hline Creatinine & 0.081 & 12.282 & & 0.485 & 2.063 \\
\hline Triglyceride & 0.795 & 1.258 & & 0.796 & 1.257 \\
\hline Lymphocyte percentage & 0.117 & 8.521 & & 0.118 & 8.494 \\
\hline Monocyte percentage & 0.336 & 2.979 & & 0.336 & 2.974 \\
\hline Neutrophil percentage & 0.103 & 9.672 & & 0.104 & 9.644 \\
\hline
\end{tabular}

of mortality in univariate models $(P<0.1)$ were included in multivariate Cox-regression models. ROC curve was used to identify the predictive value of indices for prognosis. All analyses were performed using SPSS (version 26.0, Chicago, IL, USA), and statistical significance was set up as a two-sided $P$ value $<0.05$. The formula was used to calculate the sample size: $\mathrm{n} 1=\mathrm{n} 2=$ $\frac{\left(q_{1}^{-1}+q_{1}^{-1}\right)\left(Z_{\alpha / 2}+Z_{\beta}\right)^{2} S^{2}}{\delta^{2}}$, where $(\alpha=0.05, \beta=0.10,1-\beta=0.90$, $\sigma=8.8, S=15.9$ ). The required sample size was " 70 " for each group. Improvement of PTA by at least $30 \%$ is proposed to make a difference on the curative effect [26].

\section{Results}

\section{Baseline characteristics of patients with HBV-ACLF}

The process of study selection and exclusion was shown in Fig. 1. A total of 222 patients were evaluated. After exclusion of 51 patients who did not meet the inclusion criteria, 171 cases were enrolled. Among 171 patients who were randomized (mean age, 45.2 years; 152 (88.9\%) men), 142 (83.0\%) completed follow-up through 6 months. Table 1 shows the baseline demographic and clinical characteristics for both groups. Most patients did not apply NAs treatment when the disease onset $(69.9 \%$ in the MP group and $78.4 \%$ in the control group). Twenty-one patients (12.3\%) had undetectable HBV DNA (<100 copies/ $\mathrm{ml})$ at the baseline. There was no significant difference between MP group and control group at the baseline characteristics (Table 1).

\section{Standard treatments of HBV-ACLF}

Standard treatments include antiviral drugs, plasma exchange, anti-infection drugs, and artificial centesis. No significant difference was observed between MP group and control group for standard treatments after enrollment (Table 1). $19.3 \%$ and $12.5 \%$ of patients received intensive care therapy in MP group and control group, respectively $(P=0.173) .50 .6 \%(42 / 83)$ patients in MP group received plasma exchange and $47.7 \%(42 / 88)$ patients in control group. There was no significant difference for the use of plasma exchange between groups $(P=0.707)$.

\section{Six-month primary outcome analysis Mortality and causes-of-death analysis}

At the 6-month primary end point, 88 patients had died. $43.2 \%$ occurred before day 30, $19.3 \%$ between day 30 and day 60, 11.4\% between day 60 and day 90, and $26.1 \%$ between day 90 and 6 -months. In the primary analysis, the mortality rate of MP group and control group was $32.4 \%$ and $42.5 \%$, respectively $(P=0.0037)$ at 6 months (Fig. 2a). The mortality rate of MP group was lower than control group on 14 days, 30 days, 60 days, 90 days, 120 days, 150 days, and 180 days.

Nineteen patients died from infections, 13 from HE, 16 from HRS, 6 from gastrointestinal bleeding, 5 from multiple organ failure, and 2 from cerebral hemorrhage. No significant differences in the causes of death were observed between MP group and control group (Fig. 2b, c).

Overall, 9 patients had been liver transplanted. Liver transplantation considered statistical evaluation to be an equivalent of alive in this study. $1.5 \%(1 / 83)$ patients in the MP group received liver transplantation and $10.4 \%$ $(8 / 88)$ patients in the control group. There was no significant difference for the use of liver transplantation between groups [HR (95\% CI) $0.131(0.016-1.074) ; P=$ 0.064]. The intention-to-treat concept was applied in this study. 
b

a

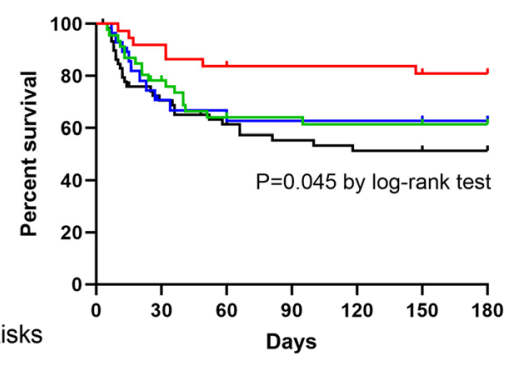

No.at Risks

$\begin{array}{llllllll}\text { MP-early ACLF } & 37 & 34 & 31 & 30 & 30 & 29 & 29\end{array}$ $\begin{array}{llllllll}\text { MP-advanced ACLF } & 46 & 36 & 28 & 26 & 25 & 25 & 14\end{array}$

$\begin{array}{llllllll}\text { Control-early ACLF } & 28 & 20 & 19 & 17 & 17 & 17 & 17\end{array}$ Control-advanced ACLF
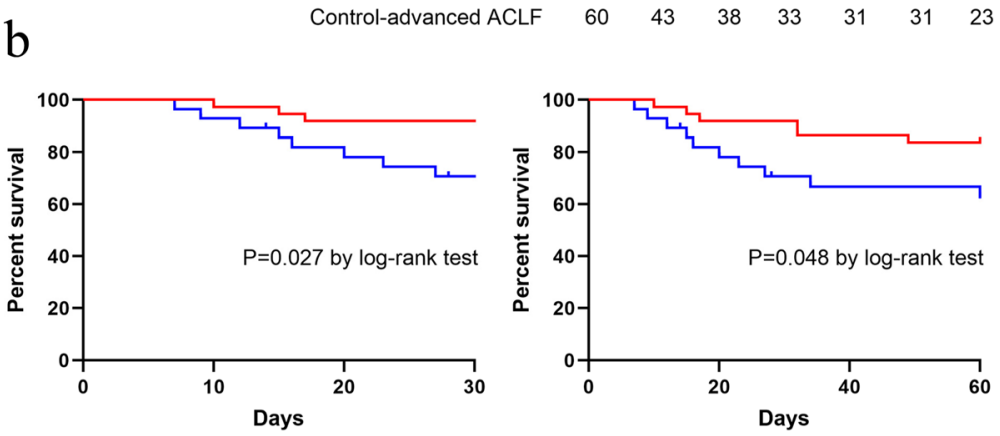

C

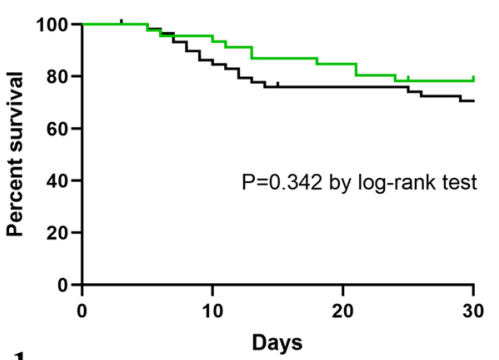

d
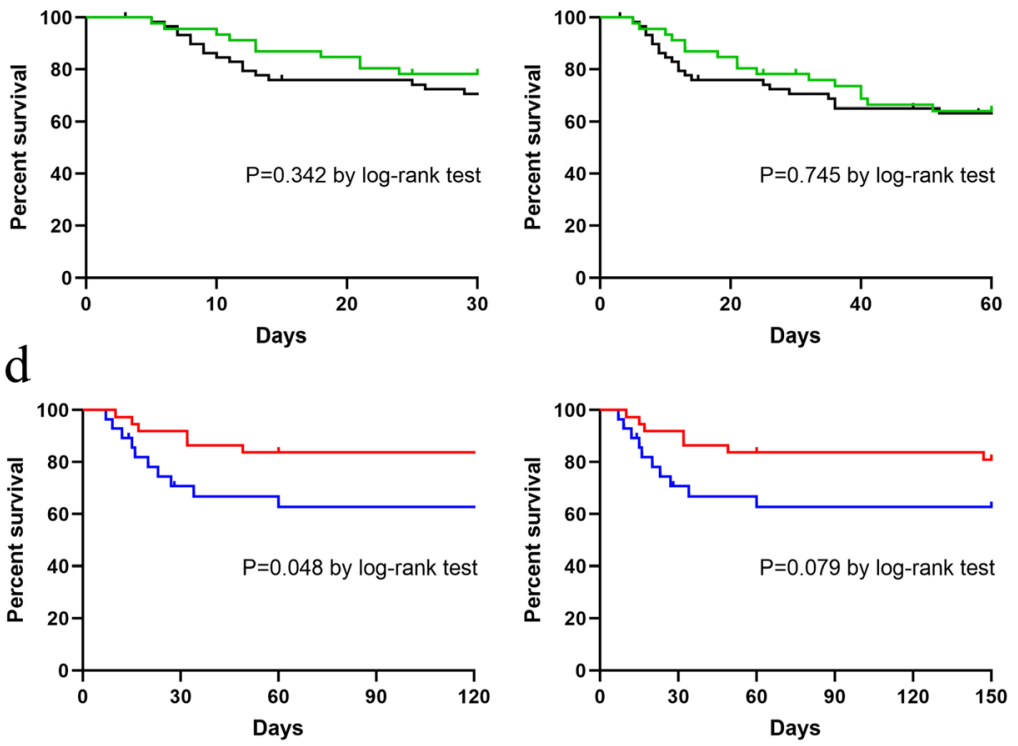

e

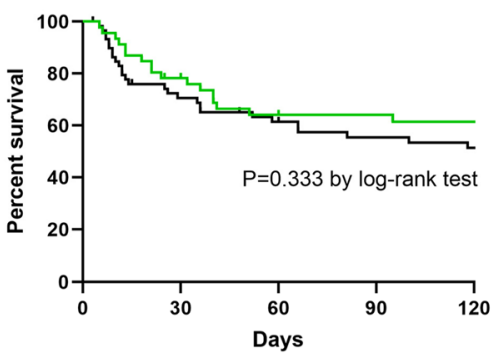

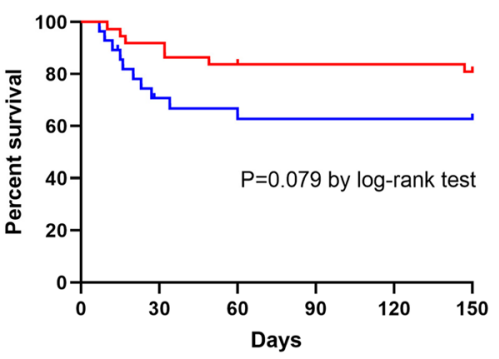

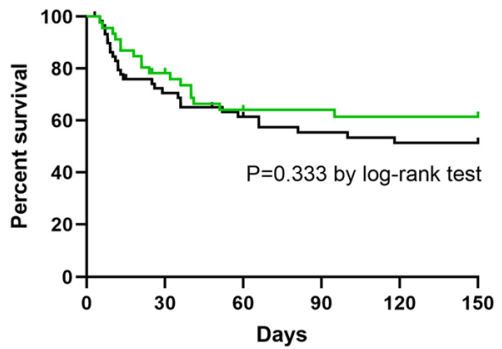

Methylprednisolone

- treated early ACLF

_. Methylprednisolone

- treated advanced ACLF

- Control-early ACLF

- Control-advanced ACLF
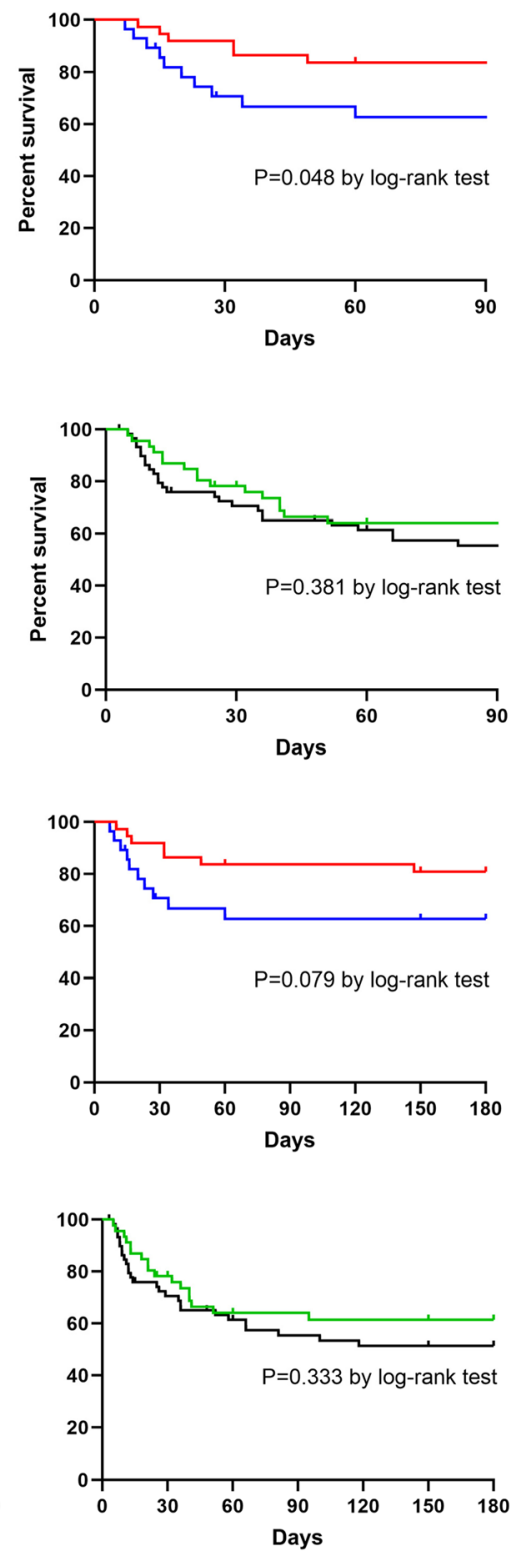

Fig. 3 (See legend on next page.) 
(See figure on previous page.)

Fig. 3 The efficacy of MP in early ACLF (those without ascites, without encephalopathy) and advanced stage ACLF. a The survival analysis of patients with ACLF in different four groups. $\mathbf{b}$ The survival analysis of patients with ACLF between methylprednisolone group and control group in early ACLF (those without ascites, without encephalopathy) during 30 days, 60 days, and 90 days. c The survival analysis of patients with ACLF between methylprednisolone group and control group in advanced ACLF during 30 days, 60 days, and 90 days. d The survival analysis of patients with ACLF between methylprednisolone group and control group in early ACLF (those without ascites, without encephalopathy) during 120 days, 150 days, and 180 days. e The survival analysis of patients with ACLF between methylprednisolone group and control group in advanced ACLF during 30 days, 60 days, and 90 days

\section{Efficacy of MP and risk factors associated with mortality at 6 months}

Based on univariate analysis and collinearity diagnosis, MP, age, bilirubin, blood urea nitrogen, creatinine, triglyceride, lymphocyte percentage, monocyte percentage, LMR, PTA, INR, ascites, HE, and HRS were included for multivariate analysis. It was shown that MP was one of the independent predictors for mortality in HBV-ACLF patient [HR (95\% CI) $0.547(0.308-0.973) ; \quad P=0.040]$ (Table 2). Meanwhile, Cox analysis revealed that HBV DNA and lymphocyte/monocyte ratio (LMR) can predict mortality of patients undergoing MP treatment $(P<0.05$, Tables 3 and 4).

The subset analysis for the efficacy of MP in those without ascites, without encephalopathy, is a useful way to show the efficacy of MP in early ACLF. Significant differences were observed $(P=0.045)$ in the survival rates among MP-treated early ACLF (81.1\%), MPtreated advanced ACLF (63.0\%), control-early ACLF (64.3\%), and control-advanced ACLF groups (55\%) at 6 months. The survival rate of MP- treated early ACLF group was higher than control-early ACLF on 30 days [HR (95\% CI) 3.981 (1.192-13.300); $P=0.027$ ], 60 days [HR (95\% CI) 2.658 (0.971-7.276); $P=0.048$ ], 90 days [HR $(95 \%$ CI $) 2.658 \quad(0.971-7.276) ; \quad P=0.048]$, and 120 days [HR $(95 \%$ CI) $2.658(0.971-7.276) ; P=0.048$ ] (Fig. 3).

\section{Six-month secondary outcome analysis Improved survival correlates with lymphocyte percentage, monocyte percentage, and LMR}

As shown in Fig. 4, the survivors had a higher lymphocyte percentage and a lower monocyte percentage in peripheral blood compared to the non-survivors at baseline $(P<0.01)$. In the MP group, the survivors had a higher LMR in peripheral blood compared to the nonsurvivors at baseline $(P<0.01)$. The MP group exhibited a rapid decrease in lymphocyte percentage, monocyte percentage, and LMR in peripheral blood during treatment compared to control group. Subsequently, the survivors in the MP group displayed a continuous increase in the above indices compared to the non-survivors $(P<$ 0.01 ). The restoration of immunity was characterized by the recovery of lymphocyte percentage, monocyte percentage, and LMR in peripheral blood after MP treatment.

Based on ROC curve, MELD+LMR had a better predictive value for prognosis of HBV-ACLF under MP treatment. The RQ cutoff value was 2.14 for LMR, 22.4 for MELD, and 0.255 for MELD + LMR (Fig. 5).

\section{Effect of MP on HBV DNA and liver function}

No significant difference in HBV DNA quantity was observed between groups $(P>0.05$, Fig. 6a). Compared to control group, serum bilirubin was lower on day 3 and day 7; PTA was higher on days $3,7,10,14,28$, and 60; INR was lower on days 7,10, and 14 in the MP group (Fig. 6b-d).

\section{Adverse events}

The incidence of hypoalbuminemia (56.6\% vs. $37.5 \%$, $P=0.012)$, fungal infection $(32.5 \%$ vs. $19.3 \%, P=$ $0.048)$, or ascites $(33.7 \%$ vs. $20.5 \%, P=0.050)$ was higher in MP group compared with the control group. Forty-seven patients developed $\mathrm{HE}, 27.7 \%$ in the MP group whereas $27.3 \%$ in the control group $(P=0.949)$. The incidence of newly onset infection was $41.0 \%$ in the MP group whereas $31.8 \%$ in the control group $(P=0.214)$. Other adverse events including electrolyte disturbance, HRS, gastrointestinal hemorrhage, acute renal insufficiency, hypoglycemia, pleural effusion, adrenal hyperplasia, and peptic ulcer were comparable between groups (Fig. 6e).

\section{Discussion}

ACLF is commonly accompanied by rapid progression, multiple organ failure, and low survival rate. Liver transplantation is the only treatment which has proven beneficial. However, the lack of donors and rapid disease progression limit its application [26, 27]. Therefore, there is an urgent need to find an effective and safe approach to ACLF. In this study, we found that MP improved the efficacy of standard treatment in HBV-ACLF, which could be a safe and effective treatment to HBV-ACLF.

The most common type of liver failure in the AsiaPacific region is HBV-ACLF. The clinical stage of HBVACLF could be divided into four stages, early ascending stage, late ascending stage, platform stage, and recovery 

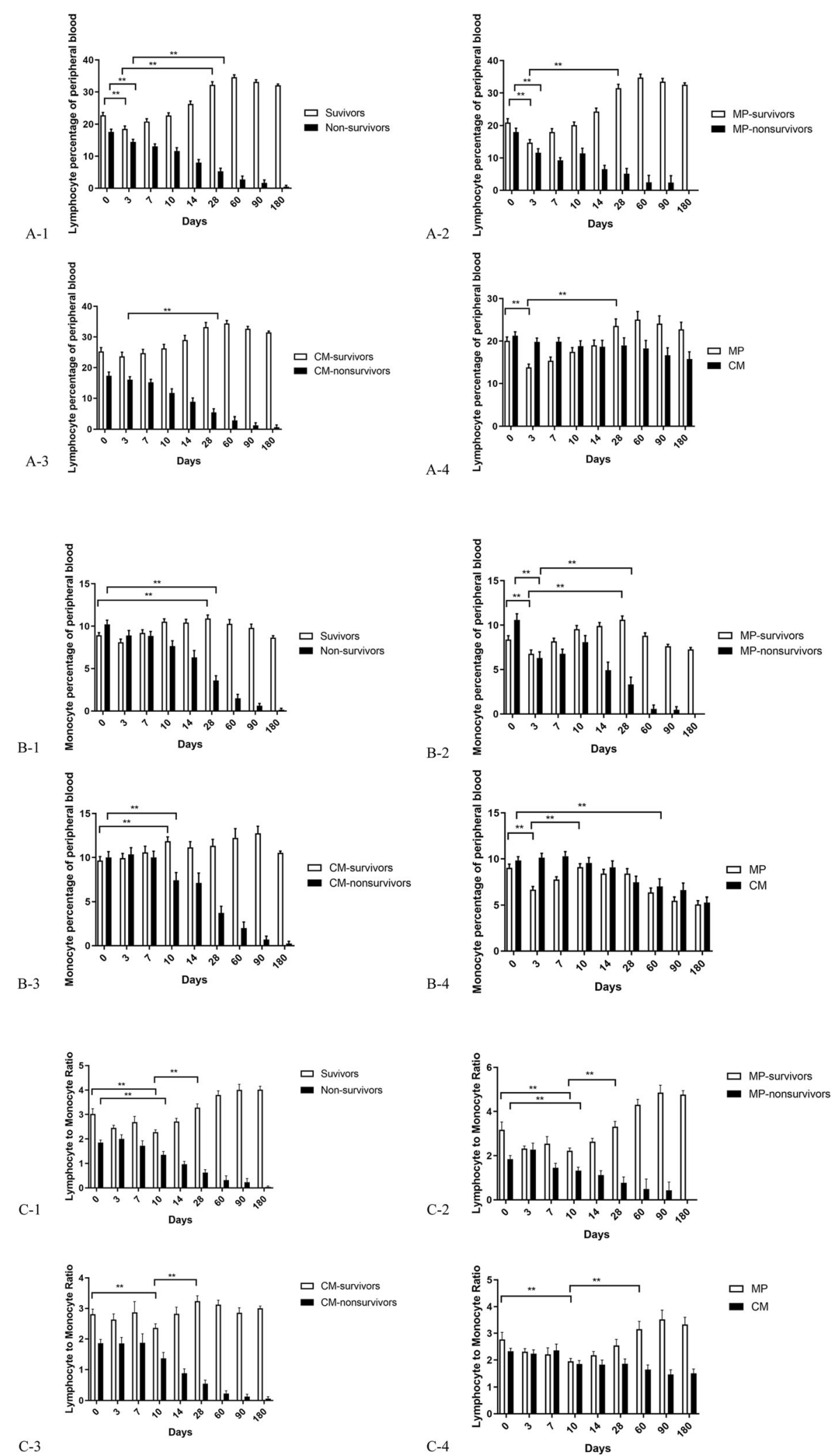

Fig. 4 (See legend on next page.) 
(See figure on previous page.)

Fig. 4 Influences of clinic indices on the outcome of HBV-ACLF. A-1 Distribution of lymphocyte percentage between survivors and non-survivors in the $171 \mathrm{ACLF}$ patients, ${ }^{* *} P<0.01$. A-2 Distribution of lymphocyte percentage between survivors and non-survivors in the MP group, ${ }^{* *} P<0.01$. A-3 Distribution of lymphocyte percentage between survivors and non-survivors in the control group, ${ }^{* *} P<0.01$. A-4 Distribution of lymphocyte percentage between MP group and control group, ${ }^{* *} P<0.01$. B-1 Distribution of monocyte percentage between survivors and non-survivors in the $171 \mathrm{ACLF}$ patients, ${ }^{* *} P<0.01$. B-2 Distribution of monocyte percentage between survivors and non-survivors in the MP group, ${ }^{* *} P<0.01$. B-3 Distribution of monocyte percentage between survivors and non-survivors in the control group, ${ }^{* *} P<0.01$. B-4 Distribution of monocyte percentage between MP group and control group, ${ }^{* *} P<0.01$. C-1 Distribution of lymphocyte to monocyte ratio (LMR) between survivors and non-survivors in the $171 \mathrm{ACLF}$ patients, ${ }^{* *} P<0.01$. C-2 Distribution of lymphocyte to monocyte ratio (LMR) between survivors and non-survivors in the MP group, ${ }^{* *} P<0.01$. C-3 Distribution of lymphocyte to monocyte ratio (LMR) between survivors and non-survivors in the control group, ${ }^{*} P<0.01$. C-4 Distribution of lymphocyte to monocyte ratio (LMR) between MP group and control group, ${ }^{* *} P<0.01$

stage [28]. Immune injury is the main event in the early ascending stage. The pathogenesis in late ascending stage is related to immune injury, ischemia, and hypoxia injury. In the platform stage, body conditions reach an immunosuppression state.
Endotoxemia is a significant factor during the initiation of liver failure. Recent studies have reported that there existed an inflammatory cascade in the early stage of HBV-ACLF [29, 30]. The earlier systemic inflammatory response syndromes (SIRS) occur, the higher

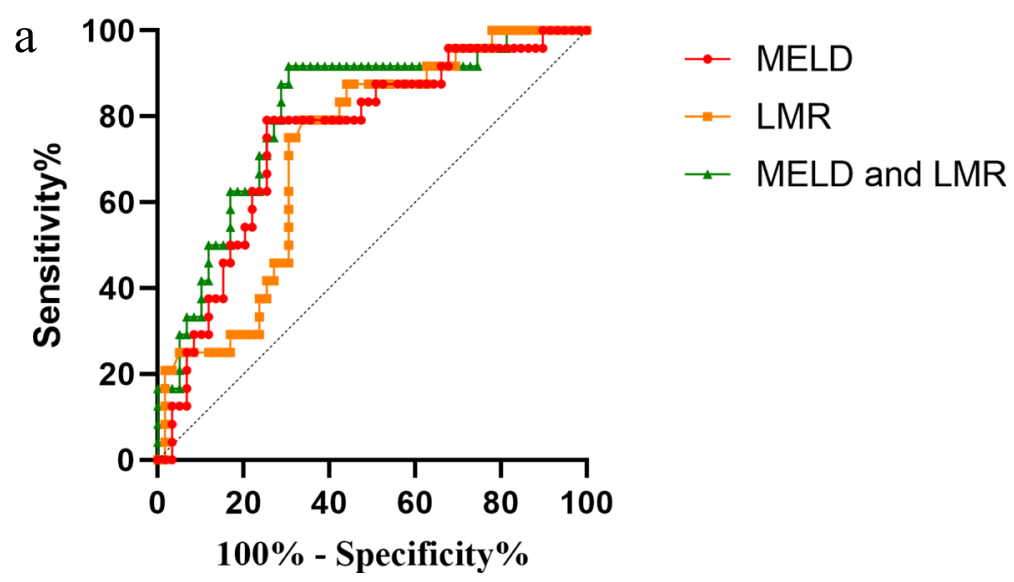

b

The predictive value of lymphocyte to monocyte ratio and MELD at baseline for clinical outcome

in HBV-ACLF patients under Methylprednisolone treatment.

\begin{tabular}{lllllllll}
\hline Factors & AUC & $95 \% \mathrm{CI}$ & $\mathrm{P}$ & $\begin{array}{l}\text { RQ } \\
\text { cutoff } \\
\text { value }\end{array}$ & $\begin{array}{l}\text { sensitivit } \\
\mathrm{y}\end{array}$ & $\begin{array}{l}\text { specificit } \\
\mathrm{y}\end{array}$ & $\begin{array}{l}\text { positive } \\
\text { predictive } \\
\text { value }\end{array}$ & $\begin{array}{l}\text { negative } \\
\text { predictive } \\
\text { value }\end{array}$ \\
\hline $\begin{array}{l}\text { lymphocyte to } \\
\text { monocyte ratio }\end{array}$ & 0.719 & $0.606-0.833$ & 0.002 & 2.14 & 0.792 & 0.661 & 0.487 & 0.886 \\
$\begin{array}{l}\text { MELD } \\
\text { Combination of }\end{array}$ & 0.751 & $0.637-0.865$ & $\begin{array}{l}<0.0 \\
01\end{array}$ & 22.4 & 0.792 & 0.746 & 0.559 & 0.898 \\
$\begin{array}{l}\text { MELD and } \\
\text { lymphocyte to } \\
\text { monocyte ratio }\end{array}$ & & & & & & & & \\
\hline
\end{tabular}

Fig. 5 a Based on ROC, predictive value of LMR+MELD was higher than any single index for mortality of methylprednisolone treatment. $\mathbf{b}$ The predictive value of lymphocyte to monocyte ratio and MELD at baseline for clinical outcome in HBV-ACLF patients under methylprednisolone treatment 

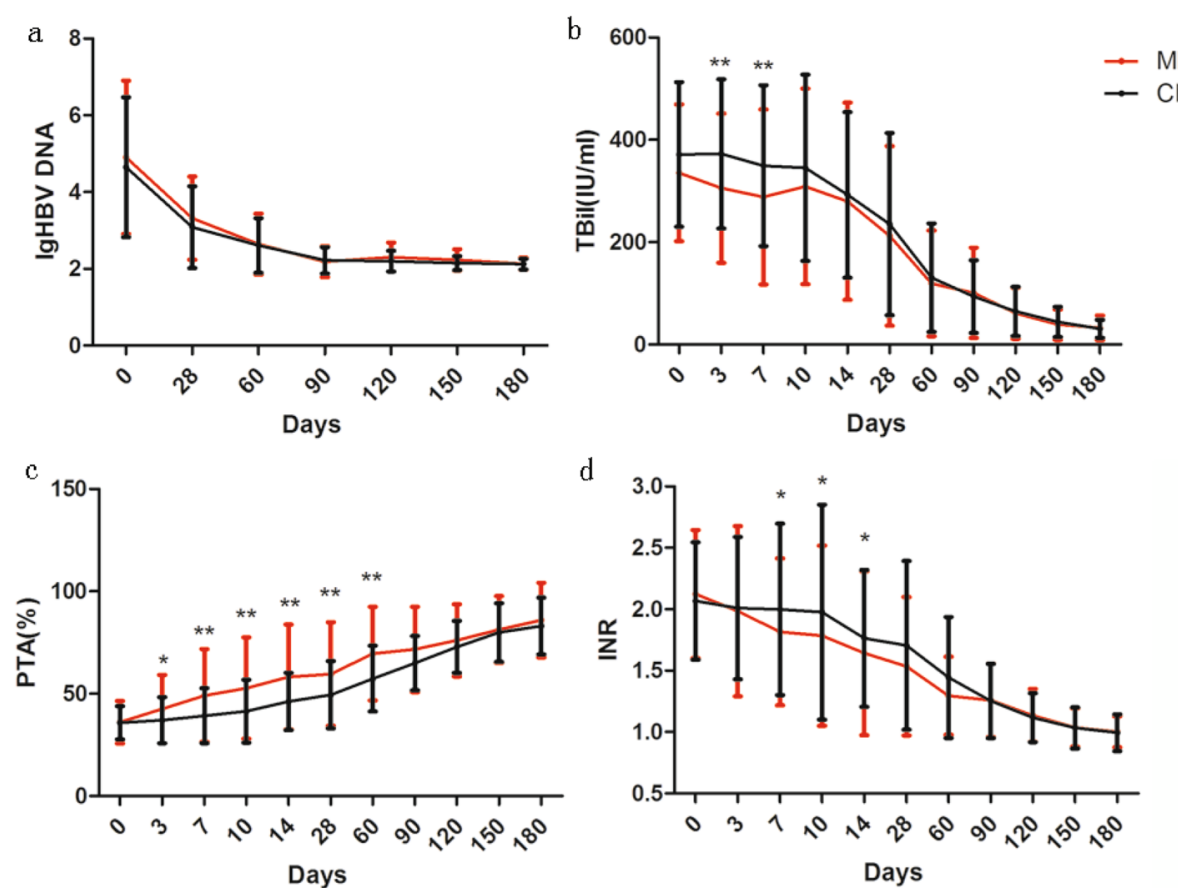

e

Adverse Events during Treatment

\begin{tabular}{llll}
\hline & $\begin{array}{c}\text { Methylprednisolone } \\
\text { group }(\mathrm{n}=83)\end{array}$ & $\begin{array}{c}\text { Control group } \\
(\mathrm{n}=88)\end{array}$ & $P$-value \\
\hline Ascites & $28(33.7 \%)$ & $18(20.5 \%)$ & 0.050 \\
fungal infection & $27(32.5 \%)$ & $17(19.3 \%)$ & 0.048 \\
Bacterial infections* & $34(41.0 \%)$ & $28(31.8 \%)$ & 0.214 \\
$\quad$ SBP & 21 & 22 & \\
$\quad$ biliary tract system & 14 & 9 & \\
$\quad$ lung infection & 8 & 4 & \\
$\quad$ enteric infection & 1 & 1 & 0.914 \\
Electrolyte disturbance & $28(33.7 \%)$ & $29(33.0 \%)$ & 0.949 \\
Encephalopathy & $23(27.7 \%)$ & $24(27.3 \%)$ & 0.012 \\
Hypoalbuminemia & $47(56.6 \%)$ & $33(37.5 \%)$ & 0.879 \\
Hepatorenal syndrome & $7(8.4 \%)$ & $8(9.1 \%)$ & 0.781 \\
Gastrointestinal hemorrhage & $3(3.6 \%)$ & $5(5.7 \%)$ & 0.353 \\
Acute renal insufficiency & $6(7.2 \%)$ & $10(11.4 \%)$ & 0.732 \\
Hypoglycemia & $2(2.4 \%)$ & $4(4.5 \%)$ & 0.623 \\
Pleural effusion & $11(13.3 \%)$ & $14(15.9 \%)$ & 0.977 \\
Adrenal hyperplasia & $1(1.2 \%)$ & $0(0.0 \%)$ & 0.503 \\
Peptic ulcer & $0(0.0 \%)$ & $2(2.3 \%)$ & \\
\hline
\end{tabular}

*one case may have more than one type of infection.

Fig. 6 Effect of methylprednisolone on HBV-DNA and indicators of liver function. No significant difference in serum HBV DNA level was observed between the two groups (a). Serum bilirubin was significantly lower on days 3 and 7 (b); PTA was higher on days 3, 7, 10, 14, 28, and 60 (c); and INR was lower on days 7 and 10 (d) in methylprednisolone group than control group. Error bars: \pm 1 SD, ${ }^{*} P<0.05$; ${ }^{* *} P<0.01$. e Adverse events during treatment

mortality would be. MP can stabilize hepatocyte membrane, suppress inflammation, and prevent further necrosis of hepatocyte [31]. Therefore, early application of
MP therapy can suppress immune response. The inhibition of systemic inflammation improves the survival rate and delays rapid progression of patients with ACLF. 
Previously, we demonstrated MP improved the 28-day survival rate in HBV-ACLF [14]. Meanwhile, our previous evidence suggested strongly that the higher myeloid $\mathrm{DC}(\mathrm{mDC})$ numbers at baseline and the recovery of $\mathrm{mDC}$ number at the end of treatment may be a prognostic marker for favorable response to MP treatment in ACLF patients. The dosage MP used in previous studies is about $1 \mathrm{mg} / \mathrm{kg} /$ day and the duration is about 3 days to 10 days [32, 33]. However, those studies were small in sample size, uncontrolled, and heterogeneous in the treatment. In our multi-center, prospective randomized controlled clinical trial, sample size is much larger than others. Meanwhile, some retrospective studies have shown that the initial dose of MP $(1 \mathrm{mg} / \mathrm{kg} /$ day $)$ did not increase adverse events or mortality [34-36]. Therefore, in order to increase the decline of the mDCs counts and make sure the recovery of $\mathrm{mDCs}$ at the end of treatment, we further increased the dosage of MP treatment, with initial dosage of MP increasing to $1.5 \mathrm{mg} / \mathrm{kg} /$ day, so as to get a stronger immunosuppressive effect at the early stage and further improve the prognosis of patients. It was observed that MP significantly decreased 6month mortality of HBV-ACLF compared to control group (32.4\% vs. $42.5 \%)$.

Mortality attributed to infection was similar across groups. Most of the adverse events in this trial were liver related. However, MP increases the incidence of fungal infection, hypoalbuminemia, and ascites. This may be related to its activation of the renin-angiotensin system and suppression of the immune response [36].

It was reported that corticosteroids may enhance HBV replication [37]. However, in our study, none of the patients on short course of MP exhibited increased HBVDNA, consistent with Zhang and Fujiwara's studies [32, 33]. MP improves liver function, possibly by preventing endotoxin-induced secondary liver injury [38], inhibiting circulating toxic substances [39], and improving the functions of remaining hepatocytes [40].

The efficacy of MP treatment is also primarily associated with the timing of MP administration [28]. In this study, due to long duration for primary care in other hospital, the patients were not timely transferred to our centers; the median time of onset for ACLF was 16 days in the MP group whereas it was 20 days in the control group. Therefore, we failed to find the significance of onset time to guide early MP therapy. It is a limitation for our study. More studies should be proceeded to explore this issue in the future. Meanwhile, age and MELD score are not well balanced between the two groups $(P=0.121 ; P=0.132)$ and it should be quoted as a limitation. Therefore, we further did propensity score matching analysis on age and MELD score to assess the baselines. It showed that Methylprednisolone group (83) could all fuzzy matched by control group (Supplementary Fig. 1).

\section{Conclusions}

In conclusion, MP therapy is an effective and safe clinical strategy in HBV-ACLF, increasing the 6-month $\mathrm{cu}$ mulative survival rate.

\section{Supplementary information}

Supplementary information accompanies this paper at https://doi.org/10. 1186/s12916-020-01814-4.

Additional file 1: Fig. S1. The propensity score matching analysis on age and MELD score to assess the baselines. It showed that

Methylprednisolone group (83) could all fuzzy matched by control group.

\section{Abbreviations}

ADV: Adefovir; AE: Acute exacerbation; AFP: Alpha-fatal protein; ALT: Alanine aminotransferase; AST: Aspartate aminotransferase; CHB: Chronic hepatitis B; ETV: Entecavir; HBV: Hepatitis B virus; HBV-ACLF: HBV-related acute-on-chronic liver failure; HBeAg: Hepatitis B virus e antigen; HBsAg: Hepatitis B virus surface antigen; HE: Hepatic encephalopathy; HRS: Hepatorenal syndrome; INR: International normalized ratio; LAM: Lamivudine; LdT: Telbivudine; LMR: Lymphocyte to monocyte ratio; MELD: Model for end-stage liver disease; MP: Methylprednisolone; NAs: Nucleoside analogs; NH3: Blood ammonia; PT: Prothrombin time; PTA: Prothrombin activity; SBP: Spontaneous bacterial peritonitis; TDF: Tenofovir

\section{Acknowledgements}

The authors are grateful for institutional support from Beijing You-An Hospital, Beijing Di Tan Hospital, and People's Liberation Army No. 302 Hospital, Beijing, China. All authors have no conflicts of interest to disclose.

\section{Authors' contributions}

Lin Jia: acquisition, analysis and interpretation of data, drafting the manuscript; Ran Xue: analysis and critical revision of the manuscript; Yueke Zhu: acquisition, analysis and interpretation of data; Juan Zhao: technical supervision; Juan Li, Wei-Ping He, Xiao-Mei Wang, Zhong-Hun Duan, Mei-Xin Ren and Hai-Xia Liu: study supervision; Hui-Chun Xing and Qing-Hua Meng: study concept and design, obtained funding, study supervision, critical revision of the manuscript for important intellectual content. All authors read and approved the final manuscript.

\section{Funding}

This work was funded by the Capital Health Research and Development of Special (2011-2018-09).

\section{Availability of data and materials}

All data generated or analyzed during this study are included in this published article.

\section{Ethics approval and consent to participate}

The research was permitted by the Ethical Committee of Beijing You-An Hospital, Capital Medical University, Beijing Di Tan Hospital Capital Medical University, and People's Liberation Army No. 302 Hospital (No.2 [2013]). All study participants gave their written informed consent to participate in the study.

\section{Consent for publication}

Not applicable

\section{Competing interests}

No potential conflict (financial, professional, or personal) is relevant to the manuscript.

\section{Author details}

${ }^{1}$ Department of Critical Care Medicine of Liver Disease, Beijing You-An Hospital, Capital Medical University, Beijing, China. ${ }^{2}$ Key laboratory of Carcinogenesis and Translational Research (Ministry of Education/Beijing), Department of phase I clinical trial, Peking University Cancer Hospital \& Institute, Fucheng Road 52, Haidian District, Beijing 100142, China. ${ }^{3} 302$ Hospital of People's Liberation Army, Liver Disease Center for Military Staff, 
Beijing, China. ${ }^{4}$ Institute of Infectious Diseases, Beijing Di Tan Hospital, Capital Medical University, No. 8 Jing Shun Dong Street, Chao yang District, Beijing 100015, China.

Received: 29 June 2020 Accepted: 13 October 2020

Published online: 08 December 2020

\section{References}

1. Sarin SK, Kedarisetty CK, Abbas Z, et al. Acute-on-chronic liver failure: consensus recommendations of the Asian Pacific Association for the Study of the Liver (APASL) 2014. Hepatol Int. 2014:8:453-71.

2. Philips CA, Sarin SK. Potent antiviral therapy improves survival in acute on chronic liver failure due to hepatitis B virus reactivation. World J Gastroenterol. 2014;20:16037-52.

3. Chen JF, Wang KW, Zhang SQ, et al. Dexamethasone in outcome of patients with hepatitis B virus-related acute-on-chronic liver failure. J Gastroenterol Hepatol. 2014;29:800-6.

4. Jalan R, Gines P, Olson JC, et al. Acute-on chronic liver failure. J Hepatol. 2012;57:1336-48.

5. Xue R, Duan Z, Liu H, et al. A novel dynamic model for predicting outcome in patients with hepatitis $B$ virus related acute-on-chronic liver failure. Oncotarget. 2017;8(65):108970-80.

6. Xue R, Zhu Y, Liu H, Meng Q. The clinical parameters for the diagnosis of hepatitis B virus related acute-on-chronic liver failure with sepsis. Sci Rep. 2019;9(1):2558

7. Claria J, Arroyo V, Moreau R. The acute-on-chronic liver failure syndrome, or when the innate immune system goes astray. J Immunol. 2016;197:3755-61.

8. Zhang Z, Zou ZS, Fu JL, et al. Severe dendritic cell perturbation is actively involved in the pathogenesis of acute-on-chronic hepatitis B liver failure. J Hepatol. 2008;49:396-406.

9. Jerala R. Structural biology of the LPS recognition. Int J Med Microbiol. 2007; 297(5):353-63.

10. Xue R, Meng Q. The management of glucocorticoid therapy in liver failure. Front Immunol. 2019;10:2490.

11. Liver Failure and Artificial Liver Group, Chinese Society of Infectious Diseases, Chinese Medical Association. Diagnostic and treatment guidelines for liver failure (2012 version). Chin J Hepatol. 2013:21(3):177-83.

12. Lee WM, Stravitz RT, Larson AM. Introduction to the revised American Association for the Study of Liver Diseases Position Paper on acute liver failure 2011. Hepatology. 2012;55:965-7.

13. Sarin SK, Kumar M, Lau GK, et al. Asian-Pacific clinical practice guidelines on the management of hepatitis B: a 2015 update. Hepatol Int. 2016;10:1-98.

14. Zhao J, Zhang JY, Yu HW, et al. Improved survival ratios correlate with myeloid dendritic cell restoration in acute-on-chronic liver failure patients receiving methylprednisolone therapy. Cell Mol Immunol. 2012;9:417-22.

15. Fujiwara K, Yasui S, Haga Y, et al. Early combination therapy with corticosteroid and nucleoside analogue induces rapid resolution of inflammation in acute liver failure due to transient hepatitis B virus infection. Intern Med. 2018;57(11):1543-52.

16. Huang $C$, Yu KK, Zheng JM, Li N. Steroid treatment in patients with acuteon-chronic liver failure precipitated by hepatitis B: a 10-year cohort study in a university hospital in East China. J Dig Dis. 2019;20(1):38-44.

17. Trepo C, Chan HL, Lok A. Hepatitis B virus infection. Lancet (London, England) 2014;384:2053-63.

18. Sarin SK, Choudhury A, Sharma MK, et al. Acute-on-chronic liver failure: consensus recommendations of the Asian Pacific association for the study of the liver (APASL): an update. Hepatol Int. 2019;13(4): 353-90.

19. Laleman W, Verbeke L, Meersseman P, et al. Acute-on-chronic liver failure: current concepts on definition, pathogenesis, clinical manifestations and potential therapeutic interventions. Expert Rev Gastroenterol Hepatol. 2011; 5(4):523-37.

20. Garcia-Tsao G. Bacterial infections in cirrhosis: treatment and prophylaxis. J Hepatol. 2005;42(Suppl):S85-92.

21. De Pauw B, Walsh TJ, Donnelly JP, et al. Revised definitions of invasive fungal disease from the European Organization for Research and Treatment of Cancer/Invasive Fungal Infections Cooperative Group and the National Institute of Allergy and Infectious Diseases Mycoses Study Group (EORTC/MSG) Consensus Group. Clinical infectious diseases: an official publication of the Infectious Diseases Society of America. Clin Infect Dis. 2008;46:1813-21.
22. Salerno F, Gerbes A, Gines P, et al. Diagnosis, prevention and treatment of hepatorenal syndrome in cirrhosis. Gut. 2007;56:1310-8.

23. Rimola A, Garcia-Tsao G, Navasa M, et al. Diagnosis, treatment and prophylaxis of spontaneous bacterial peritonitis: a consensus document International Ascites Club J Hepatol. 2000;32:142-53.

24. Liver, F., C.S.o.I.D.C.M.A. Artificial Liver Group, D. Severe liver, and C.S.o.H. C.M.A. Artificial Liver Group, [Diagnostic and treatment guidelines for liver failure (2012 version)].Zhonghua Gan Zang Bing Za Zhi, 2013, 21: $177-83$.

25. Kamath PS, Wiesner RH, Malinchoc M, et al. A model to predict survival in patients with end-stage liver disease. Hepatology. 2001;33:464-70.

26. Zhao Q, Wu CS, Fang Y, et al. Glucocorticoid regulates NLRP3 in acute-onchronic hepatitis B liver failure. Int J Med Sci. 2019;16(3):461-9.

27. Xue R, Meng Q, Li J, et al. The assessment of multipotent cell transplantation in acute-on-chronic liver failure: a systematic review and meta-analysis. Transl Res. 2018;200:65-80.

28. Dejager L, Vandevyver S, Petta I, Libert C. Dominance of the strongest: inflammatory cytokines versus glucocorticoids. Cytokine Growth Factor Rev. 2014:25(1):21-33.

29. Xing T, Li L, Cao H, Huang J. Altered immune function of monocytes in different stages of patients with acute on chronic liver failure. Clin Exp Immunol. 2007;147:184-8,

30. Chen P, Wang YY, Chen C, Guan J, Zhu HH, Chen Z. The immunological roles in acute-on-chronic liver failure: An update. Hepatobiliary Pancreat Dis Int. 2019;5:403-11.

31. Ye Y. Three attacks in the development of HBV-related liver failure. Infectious Disease Information. 2009;22:276-9.

32. Zhang $X Q$, Jiang L, You JP, et al. Efficacy of short-term dexamethasone therapy in acute-on-chronic pre-liver failure. Hepatol Res. 2011 Jan;41(1):4653.

33. Fujiwara K, Yasui S, Okitsu K, Oda S, Yokosuka O. The requirement for a sufficient period of corticosteroid treatment in combination with nucleoside analogue for severe acute exacerbation of chronic hepatitis B.J Gastroenterol. 2010; 45 (12):1255-62.

34. Arsenault RJ, Kogut MH, He H. Combined CpG and poly I:C stimulation of monocytes results in unique signaling activation not observed with the individual ligands. Cell Signal. 2013;25(11):2246-54.

35. Lin BL, Huang $Y B$, Zhang $X H$, et al. Th1/Th2 cytokine balance in patients with severe chronic hepatitis B and its relationship with prognosis. Chin J Clin Infect Dis. 2009;2:264-7.

36. Frezza G, Colli LM, De Antonio SR, De Castro M. Glucocorticoid resistance in dialysis patients reduces long-term graft survival after kidney transplantation. Transpl Immunol. 2014;30(4):145-8.

37. Liaw YF. Hepatitis viruses under immunosuppressive agents. J Gastroenterol Hepatol. 1998;13:14-20.

38. Higuchi N, Kato M, Kotoh K, et al. Methylprednisolone injection via the portal vein suppresses inflammation in acute liver failure induced in rats by lipopolysaccharide and d-galactosamine. Liver Int . 2007;27: 1342-1348.

39. Wang $M$, Shen $F$, Shi $L H$, et al. Protective effect of prednisolone on ischemia-induced liver injury in rats. World J Gastroenterol. 2008;14: 4332-7.

40. Dich J, Vind C, Grunnet N. Long-term culture of hepatocytes: effect of hormones on enzyme activities and metabolic capacity. Hepatology. 1988;8: $39-45$.

\section{Publisher's Note}

Springer Nature remains neutral with regard to jurisdictional claims in published maps and institutional affiliations. 